\title{
SOBREVOLANDO EL MUNDO DE LAS AVES: una estrategia en la enseñanza y la conservación de las aves
}

\section{Flying Over the World of Birds: A Teaching and Bird Conservation Strategy}

\author{
David Ricardo Rodríguez Villamil ${ }^{1}$
}

Fecha de recepción: 8 de febrero de 2016

Fecha de aprobación: 9 de octubre de 2016

........................................

\section{Resumen}

Este trabajo brinda información introductoria para quienes quieran desarrollar proyectos empleando las aves como un instrumento educativo, dirigido, en especial, a quienes se preocupan por la biodiversidad, las aves y la conservación.

Las aves son un diverso grupo de vertebrados y constituyen un recurso importante para la enseñanza de la biología. El presente escrito muestra el trabajo de práctica pedagógica desarrollado dentro del marco del proyecto curricular de Licenciatura en Biología de la Universidad Pedagógica Nacional, realizado durante los periodos 2013 II y 2014 I, el cual fue dirigido a estudiantes de noveno grado del Colegio Emilio Valenzuela. El objetivo de este trabajo fue construir un espacio de aprendizaje, reflexión y motivación hacia la protección y consolidación de valores en torno a la biodiversidad; así mismo, mediante el uso de binoculares, TIC (incluyendo videos, audios y grabaciones), cuaderno y diario de campo se promovió a través de la enseñanza de la ecología desde el patio de la escuela (EEPE) un acercamiento de los estudiantes hacia las aves en busca de su estudio y su conservación.

El trabajo tuvo en cuenta una serie de etapas secuenciales dispuestas en cuatro fases. Primera: identificación de aves; segunda: caracterización de ideas previas; tercera: diseño de actividades en torno a la conservación y el estudio de las aves; cuarta: aplicación de talleres teórico-prácticos (entre ellos salidas de campo), construcción de comederos y nidos artificiales.

Palabras clave: conservación, biodiversidad, aves, enseñanza-aprendizaje de la biología, nidos y comederos, salidas de campo

\section{Abstract}

This paper provides introductory information for those wishing to start in the development of educational projects using the birds as a tool to educate, directed, especially, for those who care about biodiversity, birds and conservation.

Birds are a diverse group of vertebrates, and an important resource in biology teaching. This paper shows the work of teaching practice developed within the curriculum of Bachelor's Degree of Biology at Universidad Pedagógica Nacional, held during the periods 2013-II and 2014-I, which was aimed to ninth grade students from Emilio Valenzuela school. The aim of this work was to create a learning, reflection and motivation space towards the protection and consolidation of values around biodiversity; also, using binoculars, ICT (including videos, audios and recordings), notebook and field diary an approaching of students toward the birds for their study and conservation was promoted through the Teaching Ecology from the School Yard (EEPE, for its acronym in Spanish).

1 Licenciatura en Biología, Universidad Pedagógica Nacional (Colombia). Énfasis en Biología de la Conservación. Línea de investigación: Faunística y Conservación con Énfasis en los Artrópodos. Grupo de Ornitología, Universidad Pedagógica Nacional (UPN-O), Facultad de Ciencia y Tecnología, Departamento de Biología.Correo electrónico: dbi_drodriguez104@pedagogica.edu.co 
The work considered a series of sequential steps arranged in four phases; first: bird identification; second: characterization of preconceptions; third, designing activities about birds' conservation and study; fourth: application of theoretical and practical workshops (including field trips), construction of feeders and artificial nests.

Key words: conservation, biodiversity, birds, teaching and learning of biology, nests and feeders, field trips.

\section{Introducción}

Colombia contiene una extraordinaria riqueza natural, con un aproximado de 54.871 especies biológicas encontradas en el territorio, por lo cual hace parte de los países denominados países de la megadiversidad. Así mismo, el país tiene grandes responsabilidades en la conservación y el uso correcto de los recursos naturales (Gómez, Moreno, Andrade y Rueda, 2016), y como una de las oportunidades para poder contribuir con esta responsabilidad el primer paso es una educación en conservación.

Dentro de la vasta diversidad de especies que tiene el país, este ha sido reconocido como un lugar muy importante para la observación de aves, las cuales no solo han llamado la atención de científicos, sino que, además, han maravillando a poetas, pintores, escritores, músicos, viajeros y aficionados a la observación de aves, entre otros (ABO, 2000; Renjifo et al., 2002). Actualmente, Colombia cuenta con más de 1.903 especies de aves (McMullan y Donegan, 2014), este número de especies representan cerca del $20 \%$ de todas las especies de aves del mundo y el $60 \%$ de las identificadas para Suramérica (ABO, 2000; McMullan y Donegan, 2014). Sin embargo, a pesar de esta riqueza, la biodiversidad en Colombia atraviesa momentos muy críticos, pues la desaparición de las especies va creciendo rápidamente (Renjifo et al.,2002; Renjifo et al., 2014), debido a las débiles políticas ambientales y a la explotación indiscriminada de los recursos naturales, las cuales generan graves daños a los ecosistemas que son el hogar de un sinnúmero de especies.

La diversidad de aves en un lugar refleja tanto el clima y la elevación como la disponibilidad de hábitats específicos y la estructura de la vegetación. En términos generales, la riqueza en avifauna disminuye con la elevación y es mayor en lugares húmedos que en lugares áridos. De acuerdo con ello, se esperaría que en la ciudad de Bogotá dicha diversidad fuera bastante pobre; sin embargo, en Bogotá, gracias a algunos ecosistemas como los humedales, los páramos y los bosques, todavía es posible encontrar y registrar hasta 50 especies de aves o más en una sola salida, teniendo en cuenta, además, que la Sabana de Bogotá cuenta con un registro superior a 200 especies de aves (ABO, 2000). A su vez, debido, en gran parte, a la posición geográfica de Colombia, el país recibe un flujo considerable de aves migratorias; las aves que migran de Norteamérica son llamadas migratorias boreales, mientras las que migran de Suramérica reciben el nombre de migratorias australes, y la sabana de Bogotá es un punto importante en el viaje de estas aves (ABO, 2000; Hilty y Brown, 1986; Mejía, Rueda, Galvis y Rueda, 2010).

Por lo anterior, surge la necesidad de generar procesos de enseñanza que involucren inmediatamente la diversidad biológica; en especial, por la que se halla en contextos colombianos: en primer lugar, por ser un país considerado megadiverso, y, en segundo lugar, porque esta riqueza de diversidad biológica se encuentra vulnerable y próxima a desaparecer (Acevedo, 2010).

Es así como el propósito del trabajo de práctica pedagógica buscó crear escenarios que garanticen un proceso significativo de enseñanza-aprendizaje y desarrollar un trabajo con miras a estudiar las aves reconociendo su importancia, a partir del estudio introductorio de su taxonomía, su morfo-fisiología, su evolución y su importancia ecosistémica, incluyendo la construcción de nidos y comederos artificiales, así como talleres y salidas de campo, para el acercamiento de los estudiantes a este grupo de vertebrados.

La conservación de las aves no solo es un asunto romántico justificado por el valor contemplativo o estético: éticamente, tenemos la obligación de preservar los distintos componentes biológicos que habitan la Tierra, por su derecho intrínseco a la vida, tanto como reclamamos el nuestro. Así mismo, las aves prestan importantes servicios ambientales a los ecosistemas (polinización, dispersión de semillas, agentes de control biológico y fuente de energía para otros organismos), y, por lo tanto, son consideradas especies importantes en la dinámica y el mantenimiento de los ecosistemas (ABO, 2000; Primack, 2010; Villareal et al., 2004).

\section{Maetodología}

El estudio fue dirigido a estudiantes de noveno grado del Colegio Emilio Valenzuela. En total, se contó con la participación de 87 estudiantes de tres cursos. El Colegio Emilio Valenzuela queda ubicado en el barrio San José de Bavaria, al norte de la ciudad de Bogotá, y limita con la reserva Thomas van der Hammen.

Para la realización del trabajo de práctica pedagógica fue necesario tener en cuenta una serie de etapas secuen- 
ciales, dispuestas en cuatro fases: primera: identificación de aves; segunda: caracterización de ideas previas; tercera: diseño de actividades en torno a la conservación y el estudio de las aves, y cuarta: aplicación de talleres teórico-prácticos.

\section{Primera etapa}

Para la identificación de las aves se llevaron a cabo recorridos libres alrededor del campus del Colegio Emilio Valenzuela haciendo observaciones detalladas de la avifauna presente en dicha área. Se hicieron registros visuales y auditivos empleando binoculares Bushnell $8 \times 24$, dos cámaras fotográficas Kodak 5 mm y Nikon P510, y la libreta de campo de hojas libre de ácido y rapidógrafos (como instrumentos para recopilar datos). La determinación de las especies registradas en el Colegio Emilio Valenzuela se hizo a partir de los trabajos de Hilty y Brown (1986), ABO (2000) y McMullan y Donegan (2014). La agrupación taxonómica fue desarrollada teniendo en cuenta lo planteado por el Comité de Clasificación de América del Sur (SACC, por las siglas en inglés de South American Classification Committee).

\section{Segunda etapa}

Para la caracterización de ideas previas se emplearon un taller de ideas previas y entrevistas no estructuradas, de acuerdo con Flick (2004), Cerda (2008) y Galeano (2012); así se buscó identificar los conocimientos que tenían los estudiantes acerca de las aves, además de motivarlos en la comprensión y el estudio de los problemas relacionados con la biodiversidad y su conservación.

Se realizaron entrevistas no estructuradas con el ánimo de tener un acercamiento a las ideas previas de los estudiantes. Estas consistieron en cinco preguntas:

1. ¿Cuál es el ave que ves en ese árbol?

2. ¿Cuál fue el pajarito que cantó? (En el mismo instante en el que cantaba un cucarachero común Troglodytes aedon-)

3. ¿Cuál es la importancia de la biodiversidad?

4. ¿Por qué es importante conservarla?

5. ¿Por qué en Colombia existe tanta biodiversidad?

En el mismo orden de ideas, se planteó un taller, que se desarrolló de la siguiente manera (ver Anexo 1)

La última parte de este taller consistió en mostrar fotografías de aves comunes presentes en el Colegio Emilio Valenzuela, y donde, paralelamente, los estudiantes contestaban la siguiente pregunta: ¿Cuál de las siguientes aves has observado en el Colegio Emilio Valenzuela, y qué nombre recibe? Las especies que se seleccionaron para desarrollar este punto fueron: Troglodytes aedon, Icterus chrysater, Zonotrichia capensis, Diglossa humeralis, Mimus gilvus, Molothrus bonariensis, Tyrannus melancholicus, Sicalis flaveola, Thraupis episcopus, Contopus virens y Tyrannus tyrannus, debido a su presencia en el campus del colegio.

\section{Tercera etapa}

El diseño de actividades se centró en torno a la conservación y el estudio de las aves; para ello, se consultaron algunos trabajos referentes a la enseñanza de la diversidad de aves, principalmente, mediante una búsqueda minuciosa, a partir de las bases de datos de la Universidad Pedagógica Nacional (Dialnet y Ebsco), al igual que los documentos de trabajos de grado y de práctica pedagógica del Departamento de Biología de la Universidad Pedagógica Nacional, y complementados con búsquedas en artículos científicos, libros y revistas.

Se tuvieron en cuenta para el diseño y la aplicación de actividades los siguientes trabajos:

Con el fin de crear en los estudiantes conciencia y valores hacia la conservación de la biodiversidad, Rodríguez, Rojas, Arzuza y Gonzáles (2005), quienes en el libro Loros Pericos y Guacamayas hacen un estudio de caso sobre el registro del loro orejiamarillo (Ognorhynchus icterotis), que fue reportado en 1911 como una especie abundante, pero en la década de 1980 fue declarado extinto en Colombia y Ecuador, hasta cuando fue redescubierto por el ornitólogo antioqueño Alonso Quevedo en 1998, mientras realizaba una investigación sobre la danta de montaña (Tapirus pinchaque). El libro también se tomó como referente para lecturas, ya que describe las características morfológicas de los psitaciformes, la situación actual de este grupo en Colombia, los problemas que enfrentan y algunas estrategias para su conservación a partir de la educación no formal dirigida a estudiantes y campesinos de la Región Andina.

El libro Aves de la sabana de Bogotá - Guía de campo-, de la Asociación Bogotana de Ornitología (ABO, 2000), describe los lugares, las zonas y las aves que se pueden registrar en Bogotá y sus alrededores, motivo por el cual fue tenido en cuenta para hacer salidas de campo, $y$, en general, durante todo el desarrollo del proyecto, por su valioso contenido educativo.

El proyecto Espiando aves, del Colegio Francisco José de Caldas, se revisó por tener elementos afines. El proyecto involucra los ciclos de vida de algunas de las aves de los humedales de Bogotá, como la tingua de pico rojo 
(Gallinula galeata); los temas en este proyecto fueron propuestos por los estudiantes, socializados mediante juegos y folletos, y desarrollados dentro y fuera del colegio; especialmente, en los humedales de Bogotá (Nancy Tovar, comunicación personal, 2013).

Me lo contó un pajarito es un proyecto desarrollado en el Colegio Eduardo Umaña Mendoza, de la Localidad de Usme. Busca promover el conocimiento general de las aves y un reconocimiento de las especies que habitan dicha localidad. Los estudiantes aprenden el nombre común de las aves, su nombre científico y su nombre común en inglés, y ello genera un sentido de pertenencia para con las aves y su localidad; en ese proyecto, los estudiantes comparten sus conocimientos con estudiantes de Norteamérica, quienes, en conjunto, crearon su emblema: "Tus aves también son mis aves" (Nury Penagos, comunicación personal, 2014; Penagos y Villanueva, 2013).

Así mismo, la Guía para Maestros de Escuelas de Educación Primaria, de BirdLife International (2010), da sugerencias sobre la realización de actividades educativas para desarrollar dentro y fuera del aula de clase; por su parte, el Manual para el Desarrollo y Capacitación de Guías de Aves (MacKinnon, 2004) orienta las pautas para la construcción de nidos y de comederos artificiales.

\section{Cuarta etapa}

En cuanto a los talleres teórico-prácticos, estos fueron enfocados, principalmente, a la construcción y el seguimiento de comederos y de nidos artificiales, y como parte estratégica se desarrollaron salidas de campo.

Establecer comederos es una de las mejores opciones para la observación de comportamiento en estado natural de las aves; para atraerlas, no es suficiente con arrojarles cerca algunas migas de pan, como algunas veces se piensa: todo depende de las preferencias alimentarias que tengan las aves que queremos observar. Los comederos deben instalarse cerca de algunos arbustos donde las aves encuentren refugio y seguridad. Es muy importante poner techos sobre los comederos, con el fin de prevenir que la lluvia y el sol dañen los alimentos; para efectos de este trabajo, se elaboraron comederos para aves frugívoras, nectarívoras y granívoras, utilizando materiales reciclables (BirdLife International, 2010; MacKinnon, 2004).

Igualmente, los nidos artificiales hacen una analogía con los huecos de los troncos de los árboles, los cuales son un recurso primordial para algunas aves y otros organismos; en ellos construyen sus nidos, buscan refugio y alimento y son un lugar donde establecer su progenie. Los nidos artificiales han sido utilizados en trabajos de conservación.
Un ejemplo de ello es la repoblación del loro orejiamarillo (Ognorhynchus icterotis), mediante la construcción de nidos artificiales (Rodríguez et al., 2005), y que, además, han permitido el estudio de parámetros reproductivos y de anidación, por ejemplo, de la cotorra aliazul (Hapalopsittaca fuertesi) (Tovar-Martínez, 2009).

En este sentido, la observación de las aves se ve favorecida por emplear estos instrumentos (nidos y comederos artificiales) y nos permite desarrollary agudizar los sentidos a partir de la observación, teniendo en cuenta algunos detalles que el observador debe reconocer; entre ellos:

Forma: podemos identificar un determinado grupo de aves por su forma; es decir, si, por ejemplo, es redonda y gorda, como una codorniz, o alta y delgada, como una garza.

Tamaño: para este parámetro, es necesario tener un grupo de aves conocidas que permitan la comparación con otras especies; por ejemplo: copetón, mirla o colibrí.

Postura: es decir, la posición en la que se encuentra el ave: si está vertical sobre un tronco, como lo hace un pájaro carpintero, o erecta, como un bienparado.

Coloración: es muy importante reconocer este rasgo, pues existen aves que son muy parecidas en su forma, su tamaño y su postura (condición que las agrupa en órdenes, familias, géneros y especies). Es bueno fijarse en la coloración general de las partes dorsales y ventrales, hay que prestar atención a cualquier marca o contraste que llame la atención de inmediato, como un pico rojo o una ceja.

Sonidos: muchas aves tienen cantos característicos, llamativos y diferenciables de los de otras especies, por ejemplo, el canto de un copetón, un mielero, una mirla o un toche. Igualmente, hay que prestar atención a los sonidos no vocales, como el martilleo rítmico de los pájaros carpinteros (ABO, 2000; Hilty y Brown, 1986).

También se planteó la guía de campo para ser desarrollada durante las salidas de campo en algunos de los humedales de la sabana de Bogotá (Anexo 1-2).

\section{Resultados}

Como ya se mencionó, se realizaron cuatro fases secuenciales, cada una de ellas arrojó resultados que se describen a continuación:

\section{Primera fase}

Se registraron en total 22 especies, distribuidas en 15 familias taxonómicas, como se muestra en la tabla 1 (las 
especies marcadas con * representan a las aves migratorias boreales, mientras las demás son residentes):

Tabla 1. Diversidad de aves en el Colegio Emilio Valenzuela

\begin{tabular}{|c|c|c|}
\hline Familia & $\begin{array}{l}\text { Nombre } \\
\text { científico }\end{array}$ & Nombre común \\
\hline Ardeidae & Bubulcus ibis & Garcita bueyera \\
\hline Cathartidae & Coragyps atratus & Gallinazo \\
\hline Accipitridae & Elanus leucurus & Gavilán bailarín \\
\hline Columbidae & Zenaida auriculata & Torcaza \\
\hline Trochilidae & Colibricoruscans & Colibrí chillón \\
\hline \multirow{2}{*}{ Tyrannidae } & Tyrannus melancholicus & Sirirí tropical \\
\hline & ${ }^{\star}$ Contopus virens & Pibi oriental \\
\hline Hirundinidae & Orochelidon murina & Golondrina plomiza \\
\hline Troglodytidae & Troglodytes aedon & Cucarachero común \\
\hline Turdidae & Turdus fuscater & Mirla \\
\hline Mimidae & Mimus gilvus & Mirla blanca \\
\hline \multirow{5}{*}{ Thraupidae } & Thraupis palmarum & Tangara de las palmas \\
\hline & Thraupis episcopus & Azulejo \\
\hline & Diglossa humeralis & Carbonero común \\
\hline & Sicalis flaveola & Canario criollo \\
\hline & Sicalis luteola & Canario sabanero \\
\hline Emberizidae & Zonotrichia capensis & Copetón \\
\hline Parulidae & *Setophaga petechia & Reinita amarilla \\
\hline \multirow[t]{2}{*}{ Icteridae } & Molothrus bonariensis & Chamón maicero \\
\hline & Icterus chrysater & Toche \\
\hline \multirow{2}{*}{ Fringillidae } & Spinus psaltria & Chisga \\
\hline & Spinus spinescis & Jilguero \\
\hline
\end{tabular}

Fuente: elaboración propia.

\section{Segunda fase}

La fase de caracterización de ideas previas arrojó como resultados lo siguiente:

En cuanto a las preguntas de la entrevista no estructurada:

1. ¿Cuál es el ave que ves en ese árbol? (las aves que se encontraban en los árboles eran diversas; entre ellas, tangaras). Para el $90 \%$ de los estudiantes, todas las aves eran mirlas y copetones.

2. ¿Cuál fue el pajarito que cantó? (en el mismo instante en que cantaba un cucarachero - Troglodytes aedon-). Ningún estudiante logró reconocer e identificar a qué ave pertenecía ese canto, pero sí reconocían que la escuchaban con frecuencia.

Para las preguntas 3, 4 y 5 (¿Cuál es la importancia de la biodiversidad? ¿Por qué es importante conservarla? ¿Por qué en Colombia existe tanta biodiversidad?), los estudiantes contestaron que la biodiversidad es el conjunto de animales y seres vivos, y que es importante porque nos da los recursos para sobrevivir, y por eso hay que cuidarla, si bien no supieron responder por qué en Colombia existe una megadiversidad biológica.

El análisis del taller de ideas previas arrojó como resultados:

- Para la primera pregunta se encontraron errores conceptuales, pues se definía las aves como mamíferos que tienen plumas o que las aves son artrópodos. Aunque el $60 \%$ de los estudiantes se acercaron a la definición, tan solo el $22 \%$ contestó correctamente.

- Para la pregunta 2, el $80 \%$ contestó que reconocían entre siete y diez especies de aves, mientras el $20 \%$ restante dijo no conocer más de cinco.

- En cuanto a la pregunta 3, se establecieron las siguientes categorías, con sus respectivos porcentajes de respuesta:

Número de aves presentes en Colombia, Bogotá y el CEV, respectivamente:

- Entre 1-500, 1-20 y 0-10 especies; con el 25\%.

- Entre 1000-10.000, 100-200, 16-30 especies, con el $10 \%$.

- No sabe cuántas especies hay en Colombia, Bogotá y el CEV, con el 75\%.

- Para la pregunta 4 , solo el $15 \%$ de los estudiantes contestaron adecuadamente la pregunta, al indicar que existe una variedad de preferencias en la alimentación de las aves y exponer los hábitos alimentarios de las aves carroñeras, insectívoras, polinizadoras, rapaces y granívoras. Aunque el $80 \%$ se acercaba a las descripciones, el $5 \%$ no reconoció la riqueza en los alimentos de las aves, y expusieron en este punto que no sabían de qué se alimentaban.

- En la pregunta 5 se encontró que el 5\% no sabe dónde viven e interactúan las aves; el $38 \%$ considera que los nidos son los lugares donde viven e interactúan las aves y el 57\% argumenta que los ecosistemas son los lugares propicios en los cuales las aves viven e interactúan, tanto en humedales como bosques, playas, etc. 
- La pregunta 6 consistió en responder "falso" o "verdadero":

Ningún estudiante contestó correctamente todas las preguntas. Del $100 \%$ de los estudiantes, el $45 \%$ tuvo las respuestas correctas; el 35\%, dos respuestas, y el $20 \%$ restante, solo una respuesta acertada.

- Para el punto 7, el 3\% no tuvo ninguna relación correcta; el $100 \%$ no relacionó correctamente las aves con sus respectivos alimentos; el $40 \%$ tuvo dos relaciones correctas, y el 53\% restante, solo una relación correcta.

- En cuanto al punto 8, solo se encontró que los estudiantes lograron reconocer el canto del copetón (Zonotrichia capensis).

- En el punto 9, contestaron que han observado solo tres especies: Zonotrichia capensis, Turdus fuscatery Thraupis episcopus, y que las conocen con el nombre de copetón, mirla y azulejo, respectivamente.

El taller de ideas previas y las entrevistas no estructuradas permitieron diagnosticar que los estudiantes están distantes de las aves y pocos son quienes las reconocen e identifican.
En tal sentido, surgió la pregunta de investigación que orientó el trabajo de práctica pedagógica: ¿De qué manera acercar a los estudiantes de noveno grado del Colegio Emilio Valenzuela al reconocimiento de las aves, su estudio y su conservación?

De esta manera, se continuó con la tercera y la cuarta fase del trabajo:

En estas etapas, los estudiantes diseñaron y construyeron en grupos, nidos y comederos, que fueron puestos en distintos lugares del colegio.

A los comederos inicialmente llegaron las especies Zonotrichia capensis, Zenaida auriculata, Sicalis flaveola y Thraupis palmarum. Los bebederos para colibríes fueron visitados, principalmente, por individuos de Colibri coruscans, mientras que los nidos fueron visitados por Diglossa humeralis y Troglodytes aedon.

Durante la construcción de los nidos y los comederos, los estudiantes mostraron interés y motivación por observar y estudiar la avifauna. (Figuras 1 y 2 )

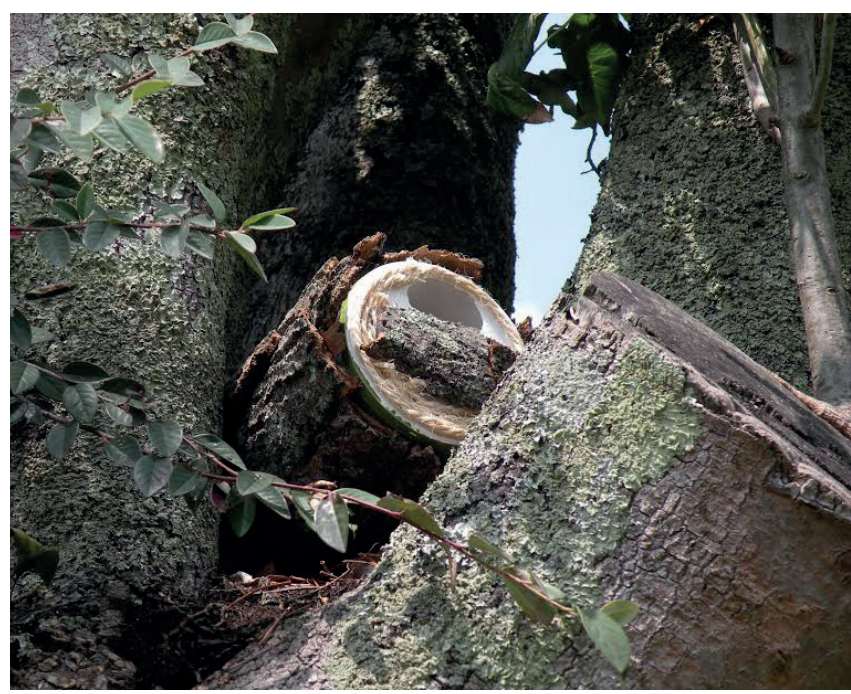

Figura 1.

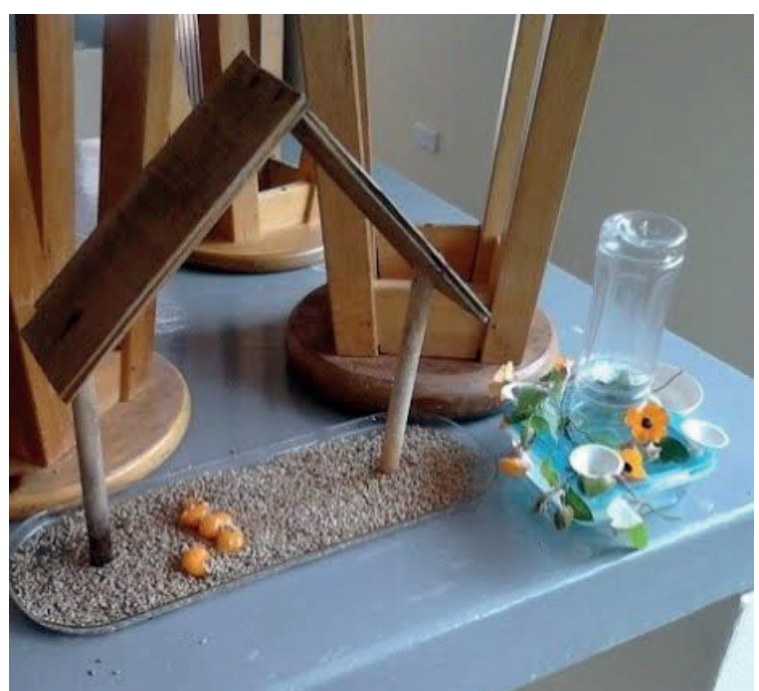

Figura 2. Construcción de nidos y comederos.
Las salidas de campo se centraron en el reconocimiento del ecosistema de humedal y las aves asociadas a dicho ecosistema, además de buscar el desarrollo de algunas destrezas científicas; entre ellas: análisis de problemas, observación, exploración, recolección y organización de información, para así permitir que se promoviesen el conocimiento y la conservación de la biodiversidad.

Las salidas de campo tuvieron lugar el 1 de agosto de 2014, y cada curso (9A, 9B y 9C) visitó un humedal: el humedal Córdoba-Niza, el humedal Santa María del Lago y el humedal La Conejera, respectivamente. Dichas visitas tenían de por medio un trabajo de campo, el cual los estudiantes desarrollaban en el transcurso del recorrido. Igualmente, se contó con el apoyo de guías de los grupos Grupo de Ornitología de la Universidad Nacional (GOUN), Aves-tamiento en Humedales y Synallaxis UD, así como el acompañamiento de los guías del Jardín Botánico de Bogotá José Celestino Mutis. 
Al finalizar la salida, el $80 \%$ de los estudiantes se mostraron sorprendidos, pues no podían creer que existieran tales ecosistemas dentro de la ciudad. Dentro de los comentarios que expresaron, está: "[...] yo todos los días paso por la Avenida Boyacá, y no me imaginaba encontrar un humedal; mucho menos, de que existieran [sic] loritos en Bogotá...deberíamos hacer estas salidas con más frecuencia" (Juan Miguel Monsalve, estudiante de 9C, comunicación personal, 2014). Ellos pasaban diariamente cerca a estos humedales y no sabían que se encontraban cerca de un ecosistema vulnerable y $\tan$ rico en biodiversidad.

Las aves que se registraron a lo largo de las salidas en los tres humedales fueron: pato turrio (Oxyura jamaicensis), pato canadiense (Anas discors), garcita bueyera (Bubulcus ibis), tingua de pico amarillo (Fulica americana), tingua de pico rojo (Gallinula galleata), mirla patiamarilla (Turdus fuscater), chamón maicero (Molothrus bonariensis), copetón (Zonotrichia capensis), chisgas (Spinus spinescens y Spinus psaltria), periquito de anteojos, o cascabelito (Forpus conspicillatus), torcaza (Zenaida auriculata), cucarachero común (Troglodytes aedon), perico de cara sucia (Eupsittula pertinax), búho negruzco (Asio stygius), correlinos (Tringa solitaria), zambullidor (Podilymbus podiceps), colibrí chillón (Colibri corunscans), pinchaflores (Diglossa humeralis y Diglossa sittoides), toche (Icterus chrysater), bababuy (Pheuticus auroventris), gallinazo (Coragyps atractus), pellar común (Vanellus chilensis), coquito (Phimosus infuscatus), gavilán bailarín (Elanus leucurus), monjita de pantano (Chrysomus icterocephalus), sirirí tropical (Tyrannus melancholicus), carpintero pardo (Picoides fumigatus), chamicero cundiboyacense (Synallaxis subpudica) y chirlobirlo (Sturnella magna). Además de las especies nombradas, en el humedal La Conejera se contó con la grata oportunidad de registrar la tingua bogotana (Rallus semiplumbeus), especie endémica de la Cordillera Oriental de Colombia, y que se encuentra catalogada como En Peligro (EN) de extinción, por el contrario, lamentablemente, no se lograron registros del cucarachero de pantano (Cistothorus apolinar) en ninguno de los humedales visitados, esta especie llama la atención porque, al igual que la tingua bogotana, es una especie endémica y se considera amenazada en la categoría de En Peligro (EN) de extinción (BirdLife international, 2000; Renjifo et al., 2002).

Cabe señalar, también, otras observaciones interesantes, entre ellas: las especies Anas discors y Tringa solitaria, fueron registradas en esta jornada de observación, siendo un registro llamativo, ya que se trata de aves migratorias boreales y es poco común encontrarlas para esta fecha; además, Anas discors fue registrado con polluelos.

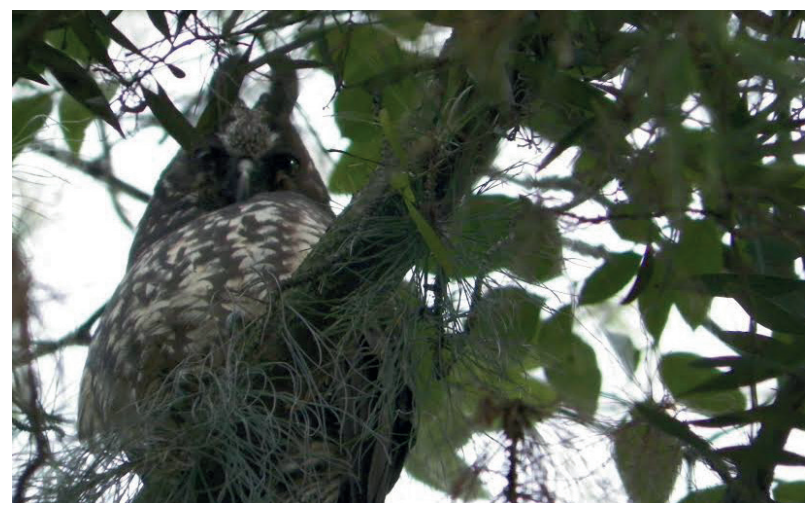

Figura 3. Búho negruzco (Asio stygius) en el humedal La Conejera.

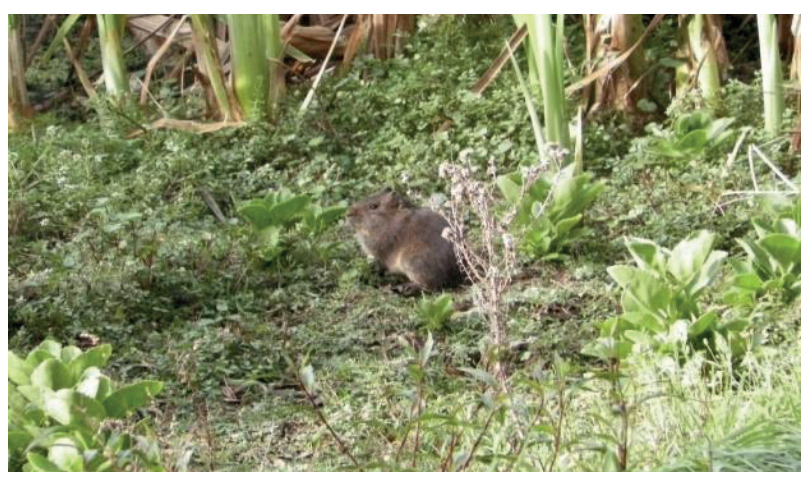

Figura 4. Curí (Cavia porcellus) en el humedal Niza-Córdoba.

Durante la ejecución del trabajo de práctica fue obligatorio el seguimiento del planeador para noveno grado de UNO-INTERNACIONAL, 2014, un libro donde el maestro y los estudiantes son los ejecutores de los contenidos, lo que dificultó en tiempo y espacio desarrollar de manera más amplia y profunda el proyecto propuesto.

En el desarrollo del proyecto se realizó un mural con el ánimo de dar a conocer el estado de los humedales como ecosistema estratégico para la conservación de las aves (figura 5):

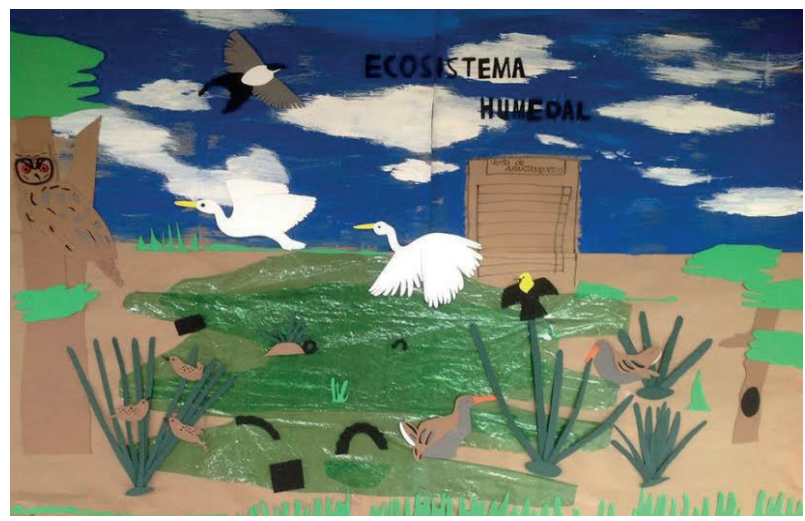

Figura 5. Mural: Los humedales, ecosistemas estratégicos para la conservación de la biodiversidad. 


\section{Conclusiones}

Es fundamental el papel que cumplen los licenciados y las licenciadas en Biología como generadores de conocimientos biológicos y pedagógicos; las aves son, a su vez, un grupo de estudio fácil de acceder, y en el cual podemos implementar la enseñanza de la biología desde el patio de la escuela, a partir del entorno inmediato, el diseño, la ejecución y la evaluación de talleres introductorios a la conservación, para ser incorporados a las actividades y los currículos escolares e institucionales (García y Torres, 2013).

Aunque faltan varios aspectos por estudiar, aprender y comprender acerca de la diversidad biológica, los licenciados y las licenciadas en Biología egresados de la Universidad Pedagógica Nacional tenemos el gran reto de enseñar la valoración, el respeto, el goce y el disfrute de nuestra afligida biodiversidad. Es urgente que incentivemos la conservación de los humedales, de las aves y de los demás componentes de la diversidad biológica que nos rodea, ya que, por ejemplo: es crítico el estado en que se encuentra el grosso de los humedales de la Sabana de Bogotá, especies de aves, tales como, el cucarachero de pantano (Cistothorus apolinari), la tingua bogotana (Rallus semiplumbeus), la tingua moteada (Porphyriops melanops), el doradito (Pseudocolpterix acutipennis), la garcita dorada (Ixobrichus exilis), la alondra cornuda (Eremophila alpestris peregrina), el búho sabanero (Asio flammeus bogotensis), y especies de aves migratorias como la reinita azul (Setophaga cerulea) y el pibí (Contopus cooperi), solo por nombrar algunas especies, se ven fuertemente amenazadas, debido a la acelerada transformación de los ecosistemas que habitan. Si no logramos tocar el corazón de las generaciones presentes y futuras, estas especies de aves en el futuro podrían, incluso, desaparecer del Distrito Capital; esto sin nombrar el estado de otros grupos faunísticos y florísticos de la ciudad y el estado en otros lugares del país.

La educación formal asume grandes retos; entre ellos, la disponibilidad de tiempo, pues el maestro debe cumplir con un currículo, un horario y unos temas específicos, y es difícil satisfacer todas las expectativas al apostarle a la educación en conservación si no se dispone de tiempo, ni del interés por parte de los estudiantes, los maestros y las instituciones educativas.
A manera de sugerencias, y de acuerdo con algunos comentarios de los profesores del Colegio Emilio Valenzuela, la implementación de comederos y de bebederos para aves podría alterar el comportamiento de estos animales; por ello, se hace urgente diagnosticar cuál es el impacto que tienen las mencionadas instalaciones en el comportamiento de las aves.

Incentivo, de manera cordial, a realizar y a apoyar otros proyectos que involucren la educación en conservación, debido a que son una punta de diamante en un mundo convulsionado y olvidado de su gran tesoro: "la biodiversidad". Invito a los lectores y a las lectoras a que desarrollen y apoyen esta clase de proyectos investigativos, ya que, con estos aportamos sustancialmente la protección de nuestra riqueza natural.

\section{Agradecimientos}

Agradezco al Colegio Emilio Valenzuela y a sus estudiantes de noveno grado, a la Universidad Pedagógica Nacional, y a la Línea de Investigación Faunística y Conservación con Énfasis en los Artrópodos, por permitir el desarrollo de la propuesta pedagógica. Al profesor Gustavo Moreno, por su disposición, su paciencia y su afán de contribuir en mi formación profesional. A los profesores Robinson Sanabria, Daniel Herrera y Eduardo Campos, por su colaboración y su orientación en el diseño y la aplicación del proyecto. A la profesora Martha García, por su disposición y sus importantes aportes. A los profesores Rodrigo Torres, Gary Stiles y Steve Hilty por su motivación y su inspiración frente al estudio y la conservación de la biodiversidad. A Gefry Andrés Castro, por las ilustraciones científicas en la guía de la salida de campo. A César Villamil y Alejandro Pérez, miembros del Colectivo Aves-Tamiento. A Liliana Andrade, del Grupo de Ornitología Synalaxis. A Jenifer Rojas y Julián Ávila, integrantes del Grupo de Ornitología de la Universidad Nacional (GOUN). Igualmente, al Jardín Botánico de Bogotá José Celestino Mutis, por su acompañamiento en las salidas de campo en los humedales visitados. Así mismo, agradezco a las personas evaluadoras anónimas, quienes hicieron interesantes aportes y sugerencias al manuscrito. 


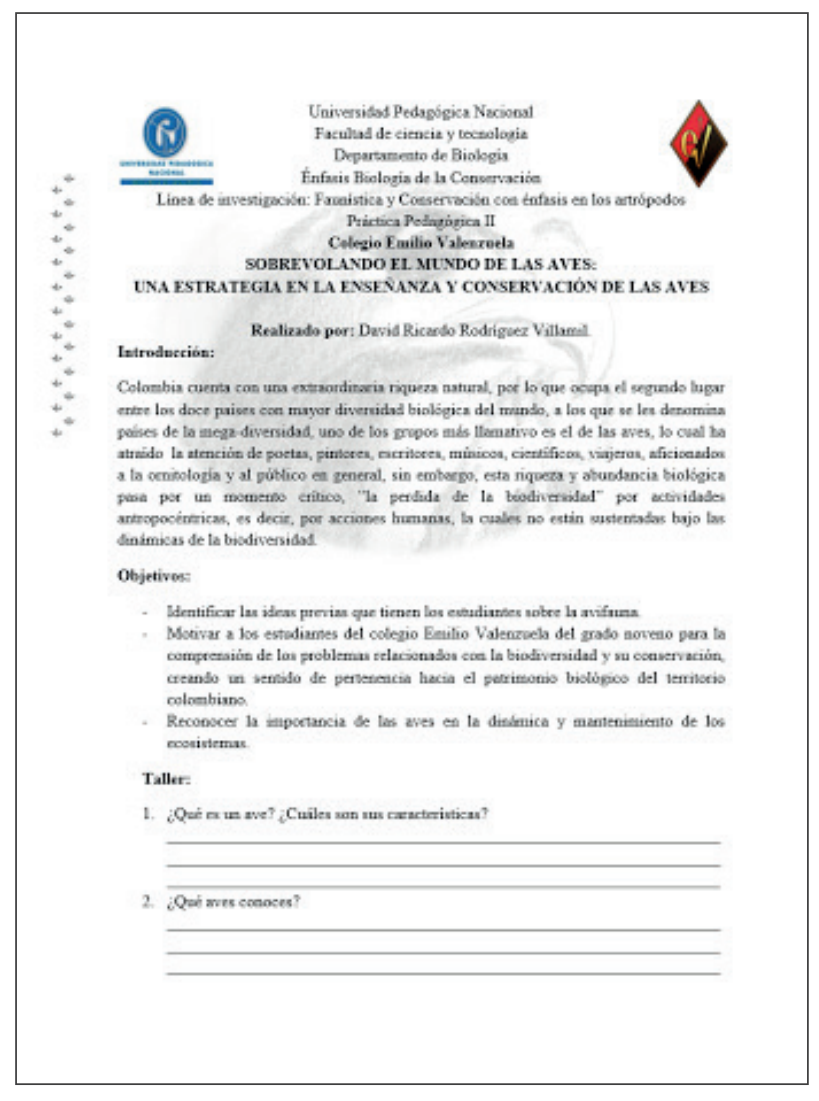

3. ¿Cuaintas especies de aves hay en tu pais, cuantas en Bogotá, cuantas en el colegio Emilio Valenzuela?

4. ¿De qué se alimentan las aves?

5. ¿En qué lugares viven las aves?

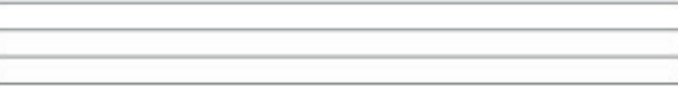

6. Responde falso (F) o verdadero (V)

Solo las aves ponen bueves ( )

Todas las aves descansan de dia ( ).

Algunas aves poseen estructuras similares a dientes ( ) .

7. Relaciona las sjouientes aves con sus alimenticios:

$\begin{array}{ll}\text { A) Colibri ( ) } & \text { A) Mosquitos } \\ \text { B) Mirla ( ) } & \text { B) Frutos y semillas } \\ \text { Paloma doméstica ( ) } & \text { C) Granos }\end{array}$

8. De los siguientes cantos escribe el nombre de la especie si la reconoces.

Especie 1

Especie 2

Especie 3

Especie 4

Especie 5

Los cantos corresponden a las siguientes especies
arysater, Trhaupis episcopus, Zonatrichía capensis y Mimus gilvus
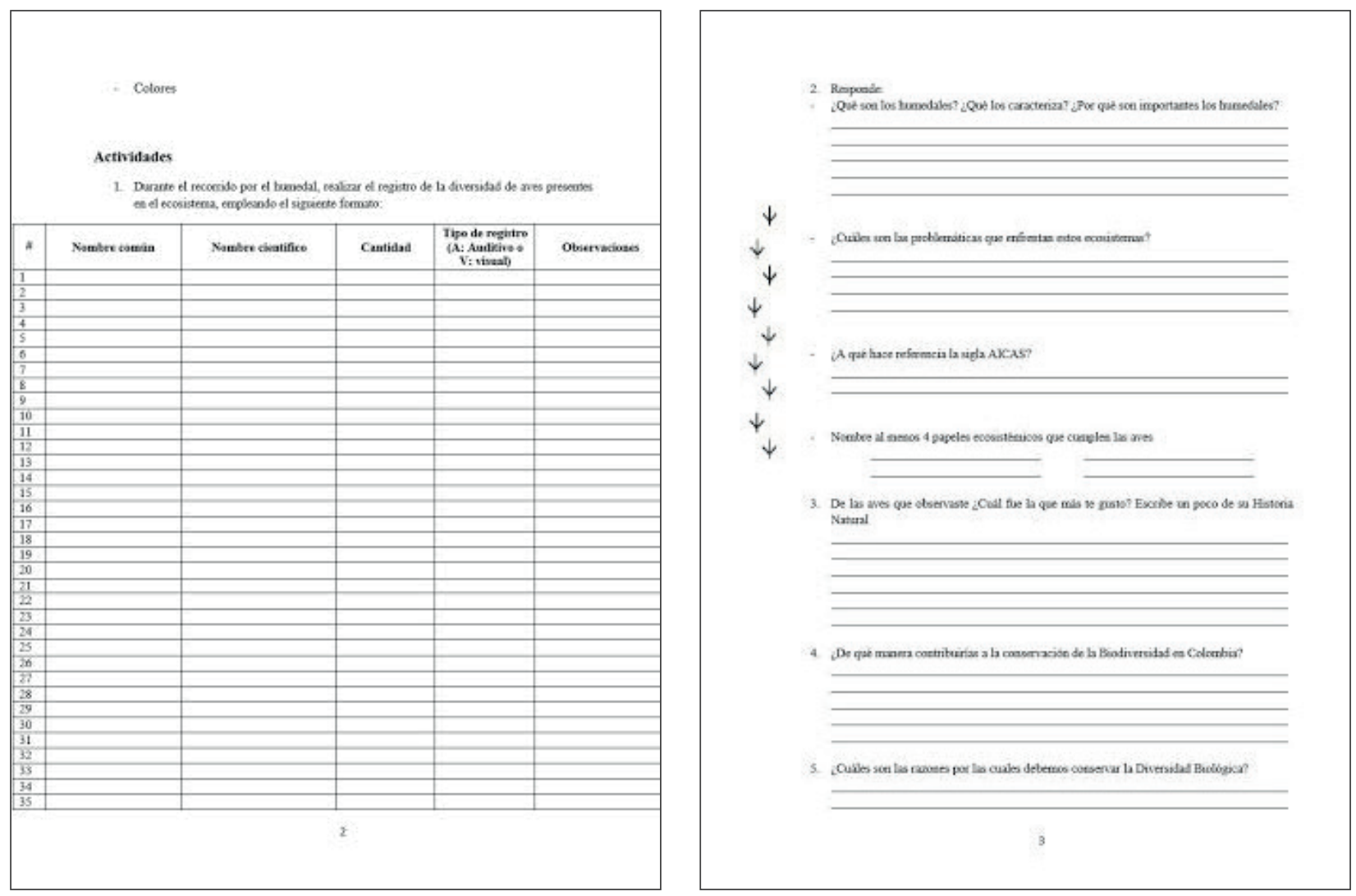

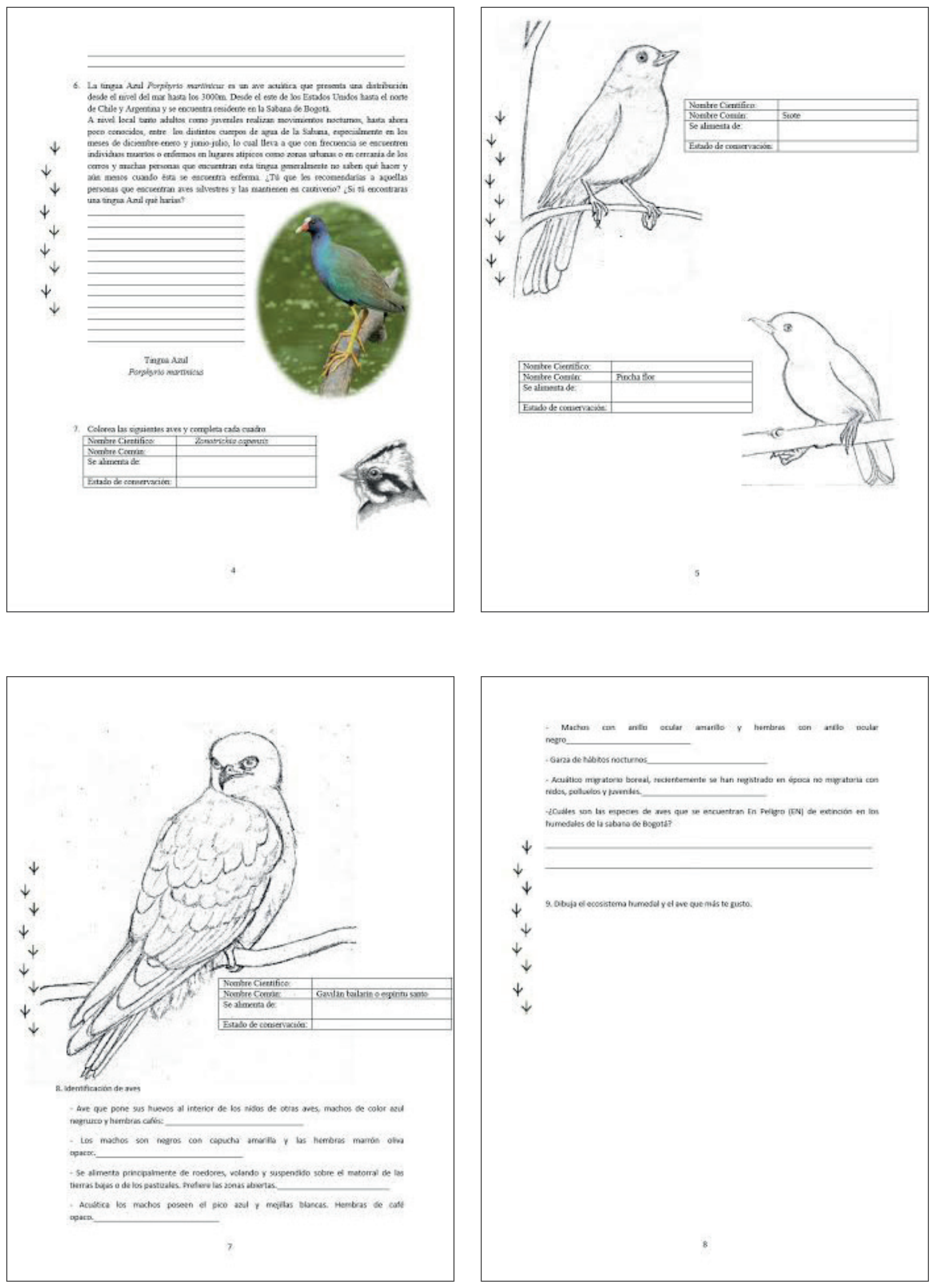


\section{Referencias}

ABO (2000). Aves de la Sabana de Bogotá: Guía de campo. $2^{a}$ reimpresión. Bogotá, D. C., Colombia: Asociación Bogotana de Ornitología y Corporación Autónoma Regional.

Acevedo, O. (2010). Algunas aves del territorio Quindicocha: valle de Sibundoy o alto río Putumayo (sudoeste de Colombia). En: G. Kattan, M. A. Echeverry-Galvis y M. López-Victoria (2013). Libro de resúmenes del IV Congreso Colombiano de Ornitología. Cali: Sello Editorial Pontificia Universidad Javeriana.

BirdLife International (2000). Threatened Birds of the World. Barcelona, España, y Cambridge, U. K.: Lynx Editions and BirdLife International.

BirdLife International (2010). Guía para Maestros de Escuelas de Educación Primaria. Recuperado en septiembre de 2013 de: http://www.birdlife.org/datazone/userfiles/file/americas/actividades_b-n.pdf

Cerda, H. (2008). Los elementos de la investigación. Bogotá: El Búho.

Flick, U. (2004). Introducción a la investigación cualitativa. Madrid: Morata.

Galeano, M. (2012). Estrategias de investigación social cualitativa. El giro en la mirada. Medellín: La Carreta.

García, M., y Torres, R. (2013). Descripción general: Línea de investigación faunística y conservación de artrópodos. Universidad Pedagógica Nacional.

Gómez, M., Moreno, L., Andrade, G. y Rueda, C. (Eds.). (2016). Biodiversidad 2015. Estado y tendencias de la biodiversidad continental de Colombia. Bogotá, D. C., Colombia: Instituto Alexander von Humboldt.

Hilty, S. y Brown, W. (1986). A guide to the birds of Colombia. Princeton: Princeton University Press.

MacKinnon, B. (2004). Manual para el Desarrollo y Capacitación de Guías de Aves. Amigos de Sian Ka'an A. C.

McMullan, M. y Donegan, T. (2014). Field Guide to the Birds of Colombia. Second edition. ProAves.
Mejía, A., Rueda, J., Galvis, P. y Rueda, J. (2010). Aves acuáticas de las ciénagas de agua dulce del departamento de Córdoba. Corporación Colombia en Hechos.

Penagos, N., y Velandia, N. (2013). Me lo contó un pajarito. Proyecto educativo del Colegio Eduardo Umaña Mendoza. Recuperado el 11 de septiembre de 2013 de http://ourbirdsareyourbirdstoo.blogspot.com.co/

Primack, R. (2010). Essentials of Conservation Biology. Fifth Edition. Sunderland, Massachusetts: Sinauer Associates, Inc.

Renjifo, L., Franco-Maya, A., Amaya-Espinel, J., Cattan, G. y López-Lanús, B. (Eds.) (2002). Libro Rojo de las Aves de Colombia. Serie Libros Rojos de Especies Amenazadas de Colombia. Bogotá, Colombia: Instituto de Investigación de Recursos Biológicos Alexander von Humboldt y Ministerio de Medio Ambiente.

Renjifo, L., Gómez, M., Velásquez-Tibatá, J., Amaya-Villarreal, A., Kattan, G., Amaya-Espinel, J., y Burbano-Girón, J. (Eds.) (2014). Libro rojo de aves de Colombia, Volumen I: Bosques Húmedos de los Andes y la Costa Pacífica. Bogotá, D. C., Colombia: Editorial Pontificia Universidad Javeriana e Instituto Alexander von Humboldt.

Rodríguez, J., Rojas, F., Arzuza, D., y Gonzáles, A. (2005). Loros, Pericos y Guacamayas. Bogotá: Conservación Internacional. Serie de Libretas de Campo.

Tovar-Martínez, A. (2009). Parámetros reproductivos y anidación de la cotorra aliazul (Hapalopsittaca fuertesi) en cavidades artificiales. Ornitología Neotropical (20), 357-368. ( The Neotropical Ornithological Society.

Villareal, H., Álvarez, M., Córdoba, S., Escobar, F., Fagua, G., Gast, F., Mendoza, H., Ospina, M. y Umaña, A. (2004). Manual de métodos para el desarrollo de inventarios de biodiversidad. Bogotá, Colombia: Programa de Inventarios de Biodiversidad. Instituto de Investigaciones de Recursos Biológicos Alexander von Humboldt. 